\title{
Magnetic Field-Induced Transformation from Paramagnetic Austenite to Ferromagnetic Martensite in an Fe-3.9Mn-5.0C (at $\%)$ Alloy*
}

\author{
By Tomoyuki Kakeshita**, Hiroo Shirai***, Ken'ichi Shimizu**, \\ Kiyohiro Sugiyama****, Kimikazu Hazumi***** \\ and Muneyuki Date****
}

\begin{abstract}
The magnetic field-induced transformation from paramagnetic austenite to ferromagnetic martensite in an Fe-3.9Mn-5.0C (at \%) alloy has been studied by means of magnetization measurement, differential scanning calorimetry and optical microscopy, applying a pulsed ultra high magnetic field. As a result, the followings were found: Transformation temperature, $M_{\mathrm{s}}^{\prime}$, shifts from the $M_{\mathrm{s}}$ temperature by a magnetic field, and the amount of the shift, $\Delta M_{\mathrm{s}}=M_{\mathrm{s}}^{\prime}-M_{\mathrm{s}}$, increases linearly with the critical magnetic field for inducing the martensitic transformation. Irrespective of $\Delta M_{\mathrm{s}}$, the amount of magnetic field-induced martensites increases with the maximum strength of magnetic field and the martensite morphology is the same as that of thermally-induced ones. A thermodynamical analysis suggests that the effect of a magnetic field on the transformation from paramagnetic austenite to ferromagnetic martensite in the Fe$\mathrm{Mn}-\mathrm{C}$ alloy is due only to the Zeeman effect, being different from that on ferromagnetic austenite to ferromagnetic martensite in other ferrous alloys, where the high magnetic field susceptibility and forced volume magnetostriction are also effective.
\end{abstract}

(Received July 2, 1987)

Keywords: martensitic transformation, magnetic field inducement, paramagnetic austenite, ferromagnetic martensite, critical magnetic field, iron-manganese-carbon alloy, martensite amount, martensite morphology, Zeeman effect

\section{Introduction}

Many studies have so far been carried out $^{(1)-(10)}$ on magnetic field-induced martensitic transformations in various ferrous alloys and steels. As a result, much useful information has been obtained about the effect of magnetic fields on martensitic transformations, such as the critical magnetic field dependency of the shift of $M_{s}$, the amount and morphology of

* A part of this paper was originally presented at the Annual Autumn Meeting of the Japan Institute of Metals, Nagoya, (1986).

** The Institute of Scientific and Industrial Research, Osaka University, 8-1, Mihoga-oka, Ibaraki, Osaka 567, Japan.

*** Graduate Student, Osaka University, at SuitaIbaraki Campus.

**** Physics Department, Faculty of Science, Osaka University, 1-1, Machikaneyama-cho, Toyonaka, Osaka 560, Japan.

***** Graduate Student, Osaka University, at Toyonaka Campus. magnetic field-induced martensites and so on. However, all of those studies have been concerned with materials undergoing a transformation from ferromagnetic austenite to ferromagnetic martensite, and no study has been made on materials exhibiting another type of transformation from paramagnetic austenite to ferromagnetic martensite. Therefore, it is not clear whether the information so far obtained is valid for the other type of martensitic transformation, and it is neccessary to confirm its validity experimentally. The $\mathrm{Fe}-\mathrm{Mn}-\mathrm{C}$ alloy may be suitable for such an experiment, because the alloy exhibiting a transformation from paramagnetic austenite to ferromagnetic martensite can easily be prepared by varying the composition of $\mathrm{Mn}$ and $\mathrm{C}$ elements, and also thermodynamics and crystallography of its thermally-induced martensitic transformation have already been well examined ${ }^{(11)(12)}$. However, the previous studies on the magnetic field-induced martensitic transformations in $\mathrm{Fe}-\mathrm{Mn}-\mathrm{C}$ alloys $^{(4)}$ did not involve considera- 
tion from such a point of view. In the present study, therefore, the magnetic field-induced martensitic transformation in an $\mathrm{Fe}-\mathrm{Mn}-\mathrm{C}$ alloy has been examined in detail by means of magnetization measurements, differential scanning calorimetry (DSC) and optical microscopy, applying an ultra high magnetic field, and compared with that in other ferrous alloys examined so far.

\section{Experimental Procedures}

An Fe-3.9Mn-5.0C (at \%) alloy was prepared by melting the component elements in a high frequency induction furnace under argon atmosphere and by casting into a watercooled iron mold. The ingot of the alloy was hot-forged at $1273 \mathrm{~K}$, homogenized at $1473 \mathrm{~K}$ for $8.64 \times 10^{4} \mathrm{~s}$ in a silica capsule filled with argon, and then quenched into iced water. Pieces of $40 \mathrm{~mm}$ length were cut from the heattreated ingot, and they were hot-rolled into $0.5 \mathrm{~mm}$ thick sheets. Specimens with a 3 $\mathrm{mm} \times 20 \mathrm{~mm} \times 0.5 \mathrm{~mm}$ size for magnetization and DSC measurements were cut from the sheets and then austenitized at $1473 \mathrm{~K}$ for $7.2 \times 10^{3} \mathrm{~s}$ in silica capsules filled with argon, followed by quenching into iced water. The austenitized specimens were chemically polished and cut into lengths of 3 and $10 \mathrm{~mm}$ by spark cutting, the $3 \mathrm{~mm}$ length specimens were used for the DSC measurements (RigakuDenki DSC 8131) to determine the $M_{\mathrm{s}}$ temperature and the $10 \mathrm{~mm}$ length specimens for the magnetization measurements. Pulsed ultra high megnetic field with the maximum strength of about $31.75 \mathrm{MA} / \mathrm{m}$ were applied to the austenitic specimens at the High Magnetic Field Laboratory of Osaka University. Details of the ultra high magnetic field instrument were described elsewhere ${ }^{(13)}$. After a magnetic field has been applied, each of the specimens was chemically etched in $35 \%$ sodium pyrosulfite solution and supplied for an optical microscopy observation. Chemical analysis of the alloy was carried out by using the rest part of the $0.5 \mathrm{~mm}$ thick sheets, which has been subjected to the same heat-treatment as above in order to avoid a discrepancy in composition between the chemically analysed and experimentally used specimens. The alloy composition thus analysed is the one mentioned at the beginning of this section.

\section{Results}

\section{Transformation temperature and magnetic property of the austenitic phase}

The DSC measurement has been made in the temperature range from 293 to $77 \mathrm{~K}$ in order to determine the transformation temperature and the latent heat of transformation. The reference material used in the measurement is the same as the present alloy, but it was subzero treated down to $77 \mathrm{~K}$ to avoid any further transformation. A typical DSC profile is shown in Fig. 1. The $M_{\mathrm{s}}$ temperature can be clearly determined to be $223 \mathrm{~K}$ for the change of heat flow. Although the $M_{\mathrm{f}}$ temperature cannot be so clearly defined, it is estimated to be about $170 \mathrm{~K}$ since there is no change in heat flow at that temperature. The latent heat of the mertensitic transformation can be obtained from the integrated value $(18.8 \mathrm{~J} / \mathrm{g})$ of heat flow in the temperature range from 170 to 223 $\mathrm{K}$ and the amount of martensites. The amount of martensites was obtained to be about $40 \%$ by the magnetization measurement in the same way as described before ${ }^{(5)}$. Using these values, the latent heat of transformation was obtained to be $2518 \mathrm{~J} / \mathrm{mol}$.

The susceptibility in the austenitic state has been obtained by the magnetization measurement, a low magnetic field (about $1.6 \mathrm{MA} / \mathrm{m}$ ) being applied. It was about $3.2 \times 10^{-13} \mathrm{H} \cdot \mathrm{m}^{2} /$ $\mathrm{kg}$ and was independent of temperature in the

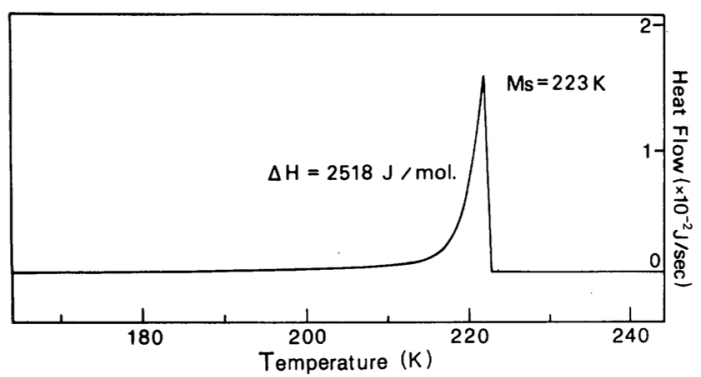

Fig. 1 DSC profile taken from an Fe-3.9Mn-5.0C (at\%) alloy in the temperature range between 77 and $293 \mathrm{~K}$. 
range from 253 to $293 \mathrm{~K}$. This means that the present $\mathrm{Fe}-\mathrm{Mn}-\mathrm{C}$ alloy is distinctly paramagnetic in the austenitic state. On the other hand, the spontaneous magnetization in the martensitic state has been obtained on the assumption that it originates in magnetic atoms and therefore it depends only upon the composition of $\mathrm{Fe}$ and $\mathrm{Mn}$ atoms. Thus, by referring to the Slater-Pauling curve ${ }^{(14)}$, the magnetic moment in the martensitic state at 0 $\mathrm{K}$ was obtained to be about $2.0 \mu_{\mathrm{B}}$ for the present $\mathrm{Fe}-\mathrm{Mn}-\mathrm{C}$ alloy, although it is very rough approximation. This value is considered to be valid in the temperature range where the magnetization measurement has been made.

\section{Critical magnetic field for inducing martensite}

Magnetization $M(t)$ has been measured as a function of magnetic field $H(t)$ in one pulse whose maximum strength of a magnetic field is higher than a critical one for inducing martensitic transformation. Typical $M(t)-H(t)$ curves at temperatures higher than $M_{\mathrm{s}}$ by 60 and $80 \mathrm{~K}$ are shown in Fig. 2, in which an increase in magnetization is recognized at a certain magnetic field as indicated by an arrow on each curve. In this way, the martensitic transformation is induced by a magnetic field even in the paramagnetic austenite, as observed in the ferromagnetic austenite ${ }^{(1)-(10)}$. Such an increase in magnetization could not be observed if the

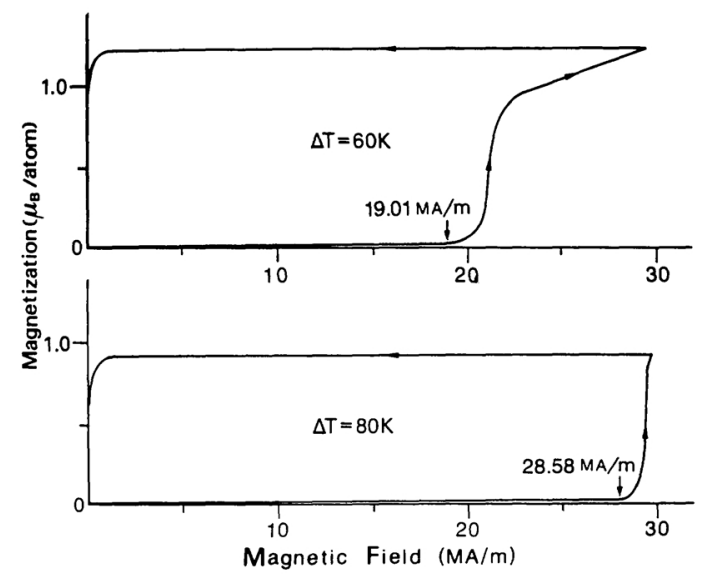

Fig. 2 Typical $M(t)-H(t)$ curves of an Fe-3.9Mn-5.0C (at\%) alloy at temperatures higher than $M_{\mathrm{s}}$ by 60 and 80 K. maximum strength of an applied magnetic field was lower than the certain magnetic field. Therefore, a certain magnetic field corresponds to the critical one for inducing martensitic transformation. The relation thus obtained between the critical magnetic field and the shift of $M_{\mathrm{s}}$ is shown in Fig. 3. It is apparent from the figure that the shift of $M_{\mathrm{s}}$ increases linearly with increasing critical magnetic field. This characteristic is very important to know the magnetic effect on transformation from paramagnetic austenite to ferromagnetic martensite. The dotted line in Fig. 3 is a calculated one which will be described later.

Another characteristic feature incidentally noted in Fig. 2 is that the high magnetic field susceptibility in the austenitic state is much smaller than that in $\mathrm{Fe}-\mathrm{Ni}$ alloys previously examined $^{(5)}$, and it is the same value as that obtained by a low magnetic field. It should also be noted in Fig. 2 that the high magnetic field susceptibility in the martensitic state is also very small. These small values of the high magnetic field susceptibility will be discussed later.

\section{Amount of magnetic field-induced martensite}

The amount of magnetic field-induced martensites has been calculated in the same manner as in the previous study ${ }^{(5)}$ by using the result of magnetization measurements. The cal-

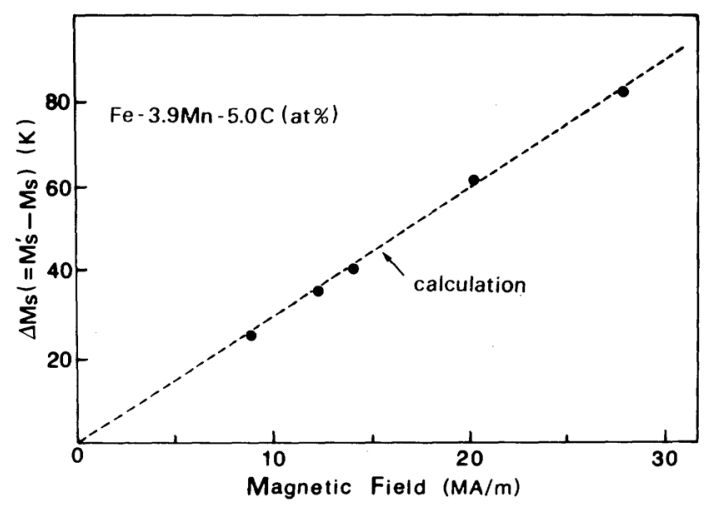

Fig. 3 Shifts of $M_{s}$ as a function of critical magnetic field for an Fe-3.9Mn-5.0C (at \%) alloy, closed circles and dotted line being measured and calculated ones, respectively. 


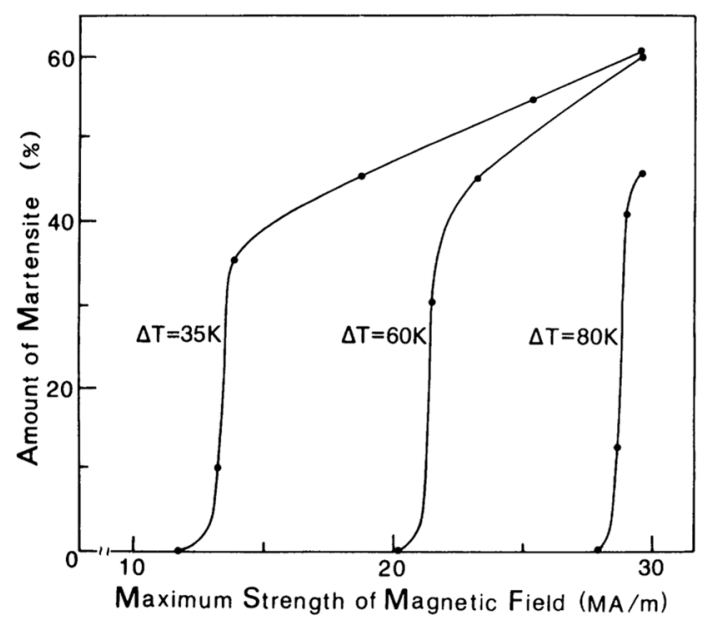

Fig. 4 Calculated amounts of magnetic field-induced martensites in $\mathrm{Fe}-3.9 \mathrm{Mn}-5.0 \mathrm{C}$ (at\%) alloy, which is plotted as a function of the maximum strength of pulsed magnetic field.

culated amount is shown in Fig. 4 as a function of the maximum strength of the pulsed magnetic field. When specimen is hold at a temperature higher than $M_{\mathrm{s}}$ by $\Delta T$, it is seen to increase with the maximum strength of magnetic field, that is, slightly near the corresponding critical magnetic field, abruptly at a little higher field than the critical one, and linearly at a much higher field. Such a magnetic field dependence of the amount of martensites is commonly observed at other temperatures of different $\Delta T$, and it is also seen in the magnetization curves in Fig. 2. The behavior relating to the increase of martensite has been examined by optical microscopy observations, as shown in Fig. 5: (a) shows a martensite structure after a magnetic field near the critical one has been applied at the temperature higher than $M_{\mathrm{s}}$ by $35 \mathrm{~K}$, which was taken at room temperature after polishing and etching; (b) shows an unetched martensite structure after a magnetic field considerably higher than the critical one has been successively applied to the same alloy at the same temperature as for (a), which was taken at room temperature for the identical place of (a). Surface relief newly appears at interfaces of martensites in (a), as known from a comparison between (b) and (a) as to the area indicated with the arrow in (a). The relief may be due to the growth of existing
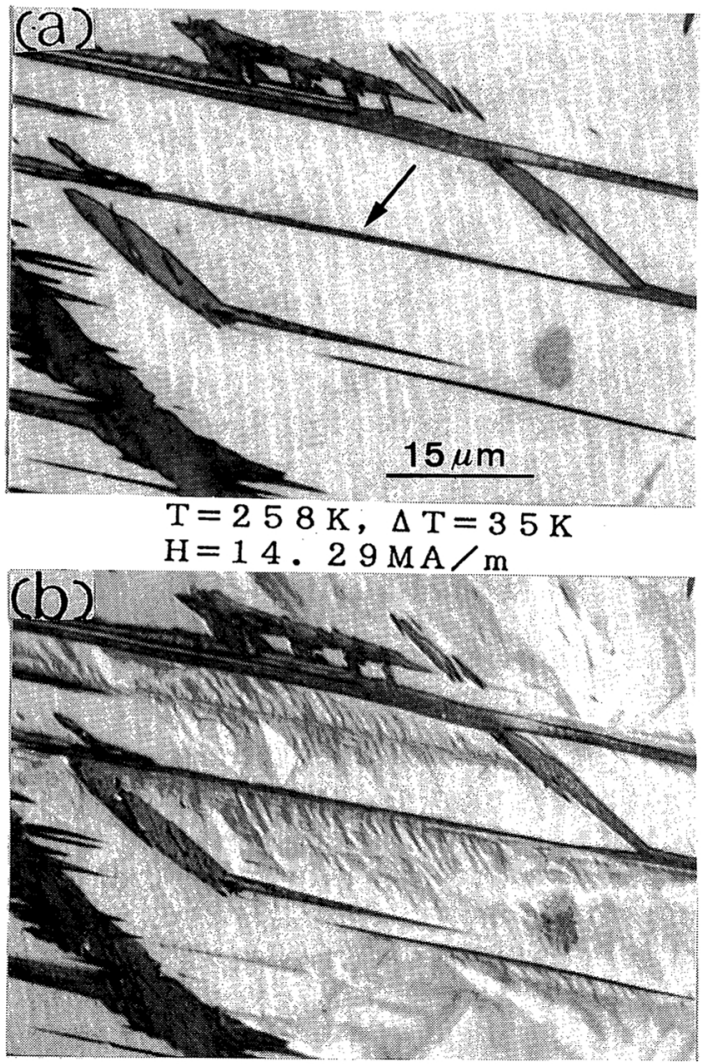

$\mathrm{T}=258 \mathrm{~K}, \Delta \mathrm{T}=35 \mathrm{~K}$

$\mathrm{H}=23.81 \mathrm{MA} / \mathrm{m}$

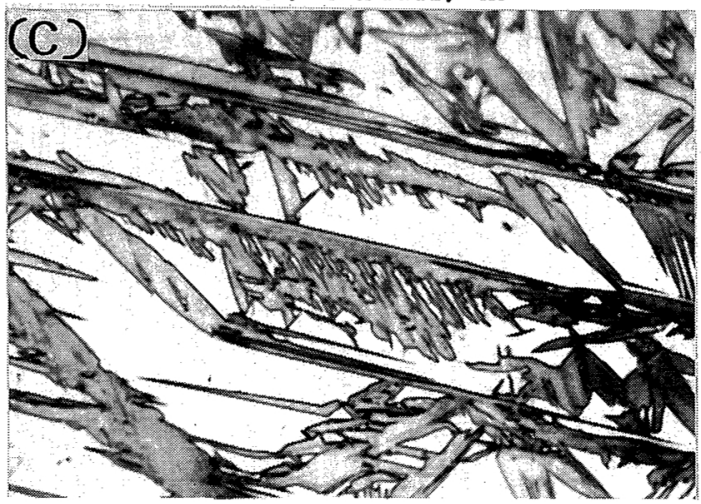

Fig. 5 Optical micrographs showing the growth of existing magnetic field-induced martensite plates and the formation of new plates in an Fe-3.9Mn-5.0C (at\%) alloy when a higher magnetic field was applied successively.

plates and/or the formation of new martensite plates. In order to make it clear, the alloy was observed after polishing and etching as shown in (c). A comparison between (b) and (c) in- 


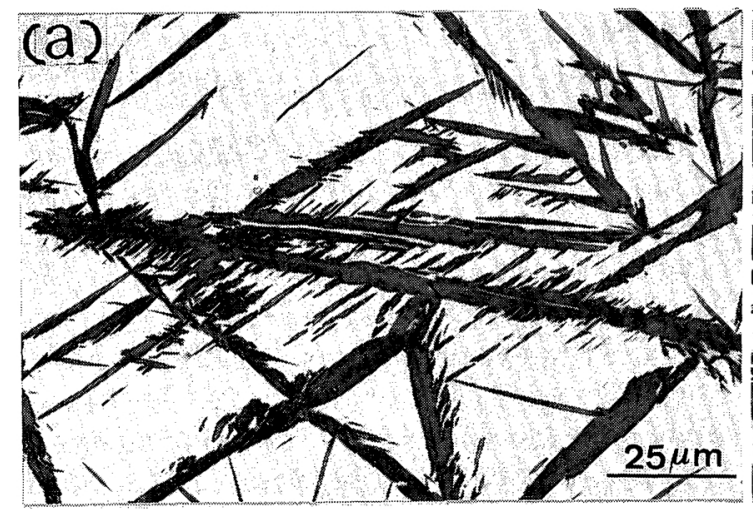

$223 \mathrm{~K}$

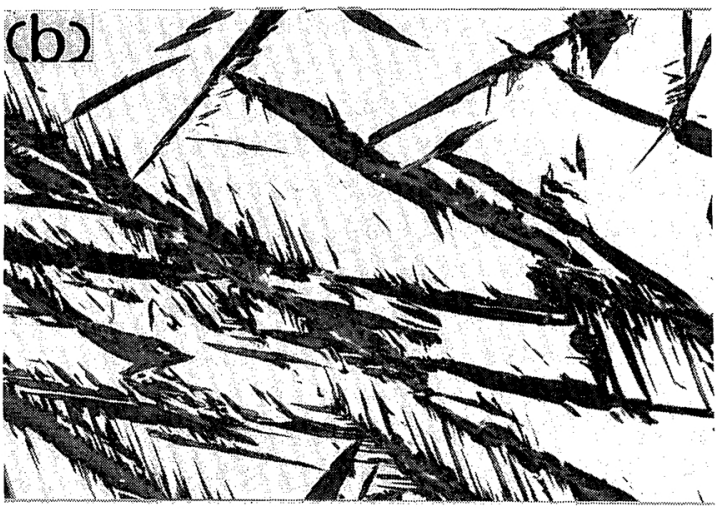

$\mathrm{T}=258 \mathrm{~K}, \Delta \mathrm{T}=35 \mathrm{~K}$ $\mathrm{H}=14.29 \mathrm{MA} / \mathrm{m}$

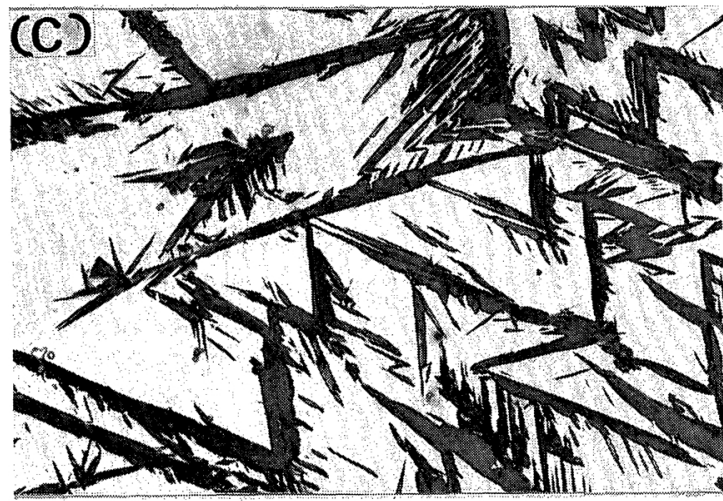

$\mathrm{T}=283 \mathrm{~K}, \Delta \mathrm{T}=60 \mathrm{~K}$

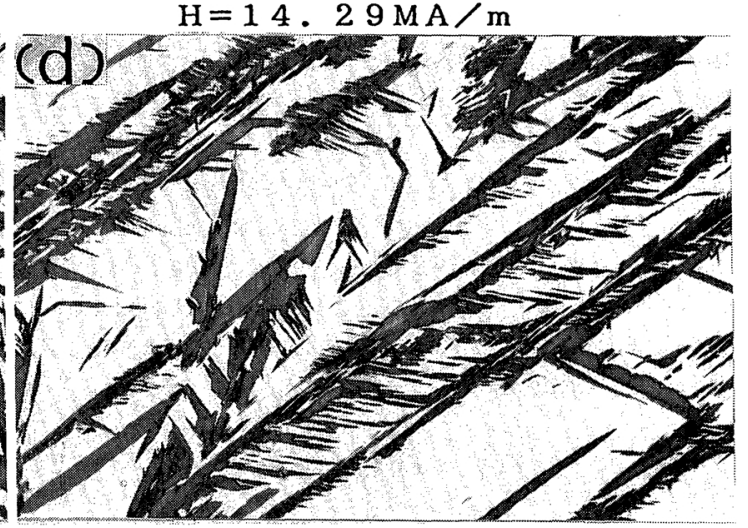

$\mathrm{H}=21.43 \mathrm{MA} / \mathrm{m}$

$\mathrm{T}=303 \mathrm{~K}, \Delta \mathrm{T}=80 \mathrm{~K}$ $\mathrm{H}=29.37 \mathrm{MA} / \mathrm{m}$

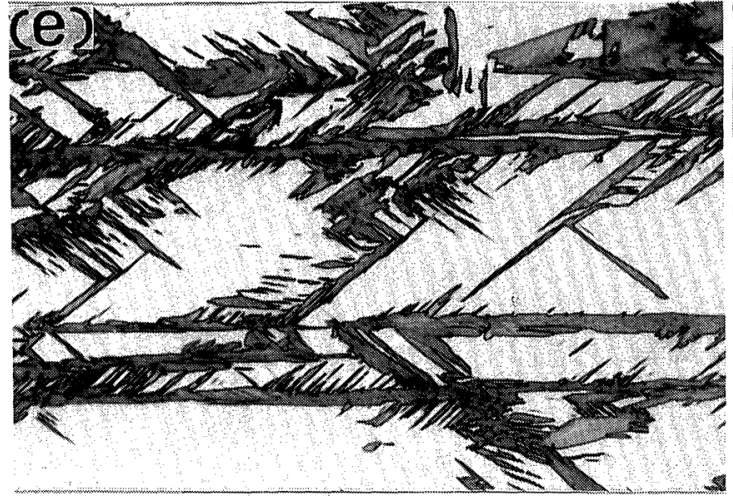

$\mathrm{T}=258 \mathrm{~K}, \Delta \mathrm{T}=35 \mathrm{~K}$

$\mathrm{H}=1.3 .49 \mathrm{MA} / \mathrm{m}$

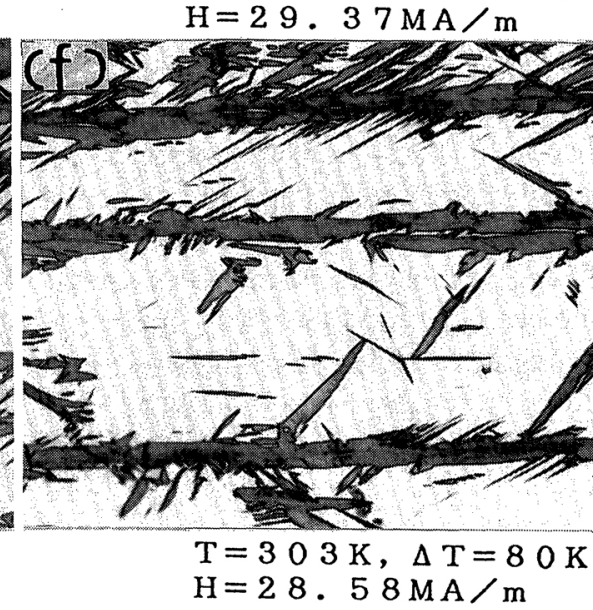

Fig. 6 Optical micrographs of thermally-induced and magnetic field-induced martensites in an Fe3.9Mn-5.0C (at \%) alloy. Transformation temperature, their difference from $M_{\mathrm{s}}$ and strength of applied magnetic field are shown for each of them.

dicates that the surface relief in (b) is caused by not only by a little growth of existing martensite plates, as arrowed in (a), but also by the formation of many small martensites.
4. Morphology of the magnetic fieldinduced martensite

Figure 6 shows optical micrographs of ther- 
mally-formed martensites formed by cooling a little below $M_{s}$ temperature, (a), and those of magnetic field-induced ones, (b) to (f). The formation temperature $T$, its difference from $M_{\mathrm{s}}$, $\Delta T$, applied magnetic field $H$ and its direction are indicated on each of the micrographs. It is to be noted in the figure that the morphology of the magnetic field-induced martensites is the same as that of thermally-induced ones irrespective of $\Delta T$. This result is the same as that of $\mathrm{Fe}-\mathrm{Ni}$ and $\mathrm{Fe}-\mathrm{Ni}-\mathrm{C}$ alloys examined previously ${ }^{(5)(8)}$. By the way, the micrographs (e) and (f) reveal that several martensite plates grow nearly parallel to the direction of applied magnetic field. This phenomenon is similar to that observed in single crystals of an $\mathrm{Fe}-\mathrm{Ni}$ alloy as apreviously examined ${ }^{(7)}$, but it is not so frequent as in the $\mathrm{Fe}-\mathrm{Ni}$ single crystals. This difference may be attributed to the fact whether grain boundaries exist or not. That is, the directional growth of martensites is suppressed by a back stress due to the existence of grain boundaries.

\section{Discussion}

It has been proposed previously ${ }^{(10)}$ that the magnetic effect on martensitic transformations is due to not only the Zeeman effect but also the high field susceptibility and forced volume magnetostriction effects. Then, relation between the critical magnetic field and the shift of $M_{\mathrm{s}}$ in the present alloy will be discussed taking into consideration the proposed magnetic effect. In the present $\mathrm{Fe}-\mathrm{Mn}-\mathrm{C}$ alloy, however, the high magnetic field susceptibility and forced volume magnetostriction may be neglected for the following reasons. The former high magnetic field susceptibility of both the austenite and martensite phases is very small, as seen from Fig. 2, and its effect on the shift of $M_{\mathrm{s}}$ is negligibly small. The forced volume magnetostriction is not effective, since the present alloy has no Invar effect because the austenite phase is paramagnetic and its lattice parameter changes normally with temperature as previously observed ${ }^{(11)}$. Thus, the proposed formula to estimate the shift of $M_{\mathrm{s}}$ of martensitic transformation under a magnetic field can be reduced as follows:

$$
\Delta G\left(M_{\mathrm{s}}\right)-\Delta G\left(M_{\mathrm{s}}^{\prime}\right)=-\Delta M\left(M_{\mathrm{s}}^{\prime}\right) \cdot H,
$$

where $\Delta G\left(M_{\mathrm{s}}^{\prime}\right)$ represents the change in Gibbs chemical free energy between the austenitic and martensitic phases at temperature $M_{s}^{\prime}$, $\Delta M\left(M_{\mathrm{s}}^{\prime}\right)$ the difference in magnetic moment between the austenitic and martensitic states at $M_{\mathrm{s}}^{\prime}$, and $H$ the critical magnetic field. The Gibbs chemical free energy of the present $\mathrm{Fe}-\mathrm{Mn}-\mathrm{C}$ alloy has been obtained by following an equation derived by Chang and $\mathrm{Hsu}^{(12)}$. The latent heat of trasformation calculated from the equation was $2522 \mathrm{~J} / \mathrm{mol}$, and this is good agreement with the experimentally determined one in the present work, $2518 \mathrm{~J} / \mathrm{mol}$, meaning that the equation is available for the present alloy system. The critical magnetic field vs $\Delta M_{\mathrm{s}}$ relation calculated by using the obtained Gibbs chemical free energy is shown by a dotted line in Fig. 3. The calculated relation is good agreement with the experimental one over the wide range of critical magnetic field. It is thus concluded that the magnetic effect exists even in materials undergoing a martensitic transformation from paramagnetic austenite to ferromagnetic martensite, but that it is due only to the Zeeman effect, being different from another transformation from ferromagnetic austenite to ferromagnetic martensite, where the high magnetic field susceptibility and forced volume magnetostriction effects are not negligible.

\section{REFERENCES}

(1) V. D. Sadovsky, N. M. Rodigin, L. V. Smirnov, G. M. Filonchik and I. G. Fakidov: Fiz. Met. Metall., 12 (1961), 302.

(2) M. K. Korenko and M. Cohen: Proc. ICOMAT-79, Cambridge, p. 388.

(3) K. R. Satyanarayan, W. Eliasz and A. P. Miodownik: Acta Metall., 16 (1968), 877.

(4) V. D. Sadovsky, L. V. Smirnov, Ye. A. Fokina, P. A. Malinen and I. P. Soroskin: Fiz. Met. Metall., 24 (1967), 918.

(5) T. Kakeshita, K. Shimizu, T. Sakakibara, S. Funada and M. Date: Trans. JIM, 24 (1983), 748.

(6) T. Kakeshita, K. Shimizu, S. Funada and M. Date: Acta Metall., 33 (1985), 1381.

(7) T. Kakeshita, S. Furikado, K. Shimizu, S. Kijima and M. Date: Trans. JIM, 27 (1986), 477.

(8) T. Kakeshita, K. Shimizu, S. Kijima, Z. Yu and M. Date: Trans. JIM, 26 (1985), 630. 
(9) T. Kakeshita, K. Shimizu, T. Maki, I. Tamura, S. Kijima and M. Date: Scripta Metall., 19 (1985), 973.

(10) T. Kakeshita and K. Shimizu: Proc. ICOMAT-86, Nara, p. 230

(11) Y. Tanaka and K. Shimizu: Trans. JIM, 21 (1980), 34.

(12) H. Chang and T. Y. Hsu: Acta Metall., 34 (1986),
333.

(13) M. Date, M. Motokawa, K. Okuda, H. Hori and T. Sakakibara: Physics in High Magnetic Fields, ed. by S. Chikazumi and N. Miura, Springer, New York, (1981), p. 44

(14) J. Grangle and G. C. Hallame: Proc. Roy. Soc., A272 (1963), 119. 